\title{
Microneedles: An Emerging Approach for Active Transdermal Delivery of Insulin
}

\section{Labiba K. El-Khordagui*}

Department of Pharmaceutics, Faculty of Pharmacy, Alexandria University, Egypt

\section{Editorial}

Since the discovery of insulin, ninety years ago, long term exogenous insulin replacement therapy has been the most effective treatment of type 1 and advanced type 2 diabetes. Because of its poor absorption, instability in the gastrointestinal tract and short half-life, insulin is conventionally administered by multiple injections, most commonly subcutaneously, to control the blood glucose level in diabetic patients. To date, a variety of injectable insulin products with varying onset and duration of action are used to meet the temporal requirements of an insulin therapy that mimics as close as possible natural production of insulin. However, pain, needle phobia and stress provoked by invasiveness and chronic parenteral therapy in addition to tissue trauma, allergic reactions and less than optimum pharmacodynamic response, have been the main limitations of subcutaneous insulin [1]. Other concerns include disposal and re-use of needles in certain communities, as a source of blood-borne pathogens. Despite improvement in needles and injection devices and infusion pumps [2], invasiveness and discomfort led in many instances to poor patient compliance and delayed insulin therapy in type 2 diabetes. Such inconveniences, on the other hand, directed developments in insulin therapy to alternative non-parenteral delivery strategies that are less invasive [3], a trend greatly enhanced by the availability of recombinant insulin on a commercial scale and a plethora of drug delivery strategies. Insulin delivery systems for oral, inhalation, intranasal, rectal or transdermal administration have been investigated with limited clinical applicability. Direct injection of insulin using a needle remains more effective and provides an avenue for effective development.

In the last decade, microneedle (MN) arrays emerged as a new transdermal drug delivery enhancement technology combining the advantages of a hypodermic needle and the convenience of a patch while reducing their limitations [4]. MN technology is based on miniaturization of multiple needles within a patch to the micron dimensions. When the patch is applied to the skin either manually or using an applicator, MNs with a length ranging from 100 to $1500 \mu \mathrm{m}$, transiently and painlessly perforate the stratum corneum (SC) having a thickness of 10-20 $\mu \mathrm{m}$ and penetrate through the epidermis to a depth of 70 to $200 \mu \mathrm{m}$. Microchannels thus created, act as temporary inskin hydrophilic pathways that facilitate delivery of small drugs such as alendronate [5], macromolecules [4,6] and nanoparticles [7] to the skin. The rich capillary bed in the dermis allows for rapid uptake of the delivered drug. Microchannels reclose within hours with recovery of skin integrity as assessed by transepidermal water loss $[8,9]$.

The design of individual $\mathrm{MN}$ arrays varies with the application. Common variables include $\mathrm{MN}$ geometric features (shape, length, diameter, aspect ratio, tip diameter and sharpness etc.), choice of fabrication material (metal, polymer, glass, ceramic, silicon, polysaccharides, etc.) and physical architecture (solid or ceramics). $\mathrm{MN}$ arrays may vary in shape, size, MNs density and interspacing, etc.
Simultaneous modulation of MNs and array variables considerably affects the device characteristics, the application force needed and the ease and cost of fabrication.

As active drug delivery devices, MNs offer great advantages such as painless and fearless drug administration, skin delivery of macromolecules and vaccines, avoidance of first-pass metabolism, possible in-skin targeting, enhanced drug efficacy with reduction in dose and side effects. Finally, modulation of drug delivery rate is possible by coupling $\mathrm{MN}$ arrays with a micropump or other physical enhancement techniques such as iontophoresis and sonophoresis [10]. On the other hand, MNs show limitations including dosage accuracy as affected by the device design, fabrication method and application to the skin, possible buckling or fracture of MNs in addition to variability of skin thickness and conditions among individuals. Such limitations have been addressed efficiently during a decade of research.

MNs can be broadly divided into two categories, solid and hollow. Solid MNs include nondissolving and dissolving/degradable MNs. Drugs can be delivered across MN-treated skin using several approaches [11]: a) "poke and patch", an approach involving skin pretreatment with solid MNs followed by topical application of drug formulation or patch on microporated skin; b) "coat and poke" where solid MNs are coated with the drug and inserted into the skin, an approach allowing precise dosing and skin administration of unstable drugs; c) "Poke and release" using drug-loaded solid MNs made of dissolving/ biodegradable polymers or polysaccharides allowing simultaneous skin microporation and drug release in one step. No patch or micropump is needed and no hazardous sharp waste left; d) "poke and flow" where hollow microneedles are used to infuse a liquid drug formulation into the skin. Hollow MNs can also be used to withdraw dermal interstitial fluid for glucose monitoring [4].

MN fabrication is based on microfabrication methods utilizing photolithography, silicon etching, laser micromachining, micromolding and microelectromechanical (MEMS) techniques [4,11]. Selection of MN category, fabrication material and method as well as the drug delivery approach is application-specific. It depends on the drug molecular characteristics, the dose and whether local intradermal or transdermal delivery is required.

*Corresponding author: Labiba K. El-Khordagui, Department of Pharmaceutics, Faculty of Pharmacy, Alexandria University, Egypt, E-mail: lakhalil@alexpharmacy.edu.eg

Received October 28, 2012; Accepted November 06, 2012; Published November 10,2012

Citation: El-Khordagui LK (2012) Microneedles: An Emerging Approach for Active Transdermal Delivery of Insulin. J Bioequiv Availab 4: xxxi-xxxiii. doi:10.4172/ jbb.10000e24

Copyright: () 2012 El-Khordagui LK. This is an open-access article distributed under the terms of the Creative Commons Attribution License, which permits unrestricted use, distribution, and reproduction in any medium, provided the original author and source are credited. 
Citation: El-Khordagui LK (2012) Microneedles: An Emerging Approach for Active Transdermal Delivery of Insulin. J Bioequiv Availab 4: xxxi-xxxiii. doi:10.4172/jbb.10000e24

As macromolecules are target candidates of $\mathrm{MN}$-driven transdermal delivery, a great deal of MN-based research has been focused on macromolecular therapeutics with insulin receiving most attention. Proof of concept was followed by progressive improvement using different types of MNs and combined approaches. Preclinical studies using solid MNs for skin pretreatment demonstrated significant increase in delivery of insulin solution and reduction in blood-glucose level [12]. The technique was improved by using MN rollers [13], lyophilized hydrogel insulin patch to replace the insulin solution [14] and by combining MN pretreatment with iontophoresis and insulinloaded nanovesicles [15]. Simultaneous basal-bolus delivery of fastacting insulin could also be achieved by pretreating the skin of diabetic rats with short solid MNs $(150 \mu \mathrm{m})$ and applying an iontophoresis transdermal patch with insulin reservoir for on-demand iontophoresistriggered dose for postprandial insulin administration [16]. Solid MNs coated with insulin proved to be an alternative single step means for effective insulin delivery [17].

Further, solid MNs of the dissolving/biodegradable type encapsulating insulin, fabricated under mild temperature conditions and circumventing the use of organic solvents were shown to preserve insulin stability and bioactivity and to enable effective delivery upon insertion into the skin [18]. Attempts were made to prolong pharmacodynamic effects in rats by using intermediate-acting insulin [19].

Hollow microneedles present an alternative MN type for insulin delivery via the "poke and flow" approach. They offer advantages over solid MNs by allowing faster active delivery of the drug solution by combining a microneedle injection applicator with a syringe, a handpressed flexible drug reservoir, an iontophoresis unit, a micropump or a microfluidic chip [11]. In clinical studies, hollow MNs were more convenient compared to conventional subcutaneous catheters. In addition, hollow MN-driven intradermal insulin delivery induced faster insulin absorption and enabled more rapid onset and offset of metabolic effect on blood glucose levels which was attributed in larger cohorts of type I diabetic patients to rapid lymphatic uptake and distribution of insulin from the skin [20]. Hollow MNs allow the delivery of a larger amount of drug compared to other MN types and hence have been the subject of a large proportion of patents filed based on MN type and clinical studies of insulin delivery to type 1diabetes subjects [21].

Extensive research over the last decade provided ample proof of the potentials of $\mathrm{MN}$ arrays with optimized characteristics for the active dermal delivery of insulin and reduction of blood sugar level in a non-invasive painless manner. Clinical findings coupled with patient acceptability and possibility of self-administration put forward MN technology as a platform for an alternative insulin therapy for millions of diabetic patients worldwide, including pediatric and geriatric patients, assuming cost effectiveness.

For effective exploitation of MN technology in the management of insulin therapy, complex interdependencies of technological, pharmaceutical and biological variables involved in the $\mathrm{MN}$-insulin formulation-patient interaction warrant multidisciplinary research and development initiatives. Quality attributes of an ideal MN system for diabetes management will be governed by advances in biomedical engineering, microelectronics and microfluidics. At the broadest level, a prototype MN-based device for diabetes management is an autonomous $\mathrm{MN}$-based automated device whereby a glucose sensor as a diagnostic component is connected with a therapeutic component consisting of a hollow MNs array/micropump for the intermittent active dermal delivery of insulin. Both components are bridged by a feedback microchip which regulates the blood glucose level by adjusting insulin delivery. Such a system allows for accurate patient-specific tailoring of the glucose level versus quantitatively and temporally controlled insulin delivery [22]. However, for years to come, stand-alone glucose sensors and insulin delivery systems will be used by diabetic patients with $\mathrm{MN}$ technology providing a future patient friendly therapeutic modality.

Finally, in order to move MNs to the market, specific regulatory guidelines for the development of different microneedle systems should be set by regulatory authorities. Conformation to such guidelines and to quality standards of drugs and bioactive agents will safeguard efficacy and safety of insulin/MN devices. Acceptability of these devices by diabetic patients, long-cherishing a painless insulin therapy, will be maximized by price affordability and evidence for cost effectiveness.

\section{References}

1. Sabetsky V, Ekblom J (2010) Insulin: a new era for an old hormone. Pharmaco Res 61: 1-4

2. Nitesh S, Sanjeev C, Vandana H, Alka A, Vijender S (2011) Recent Advances in Insulin Delivery Systems: An Update. World Appl Sci J 11: 1552-1556.

3. Khafagy El-S, Morishita M, Onuki Y, Takayama K (2007) Current challenges in non-invasive insulin delivery systems: a comparative review. Adv Drug Deliv Rev 59: 1521-1546.

4. Kim YC, Park JH, Prausnitz M (2012) Microneedles for drug and vaccine delivery. Adv Drug Del Rev 64: 1547-1568.

5. Katsumi H, Liu S, Tanaka Y, Hitomi K, Hayashi R, et al. (2012) Development of a novel self-dissolving microneedle array of alendronate, a nitrogencontaining bisphosphonate: evaluation of transdermal absorption, safety, and pharmacological effects after application in rats. J Pharm Sci 101: 3230-3238.

6. Gomaa YA, Garland MJ, Mclnnes F, El-Khordagui LK, Wilson C, et al. (2012) Laser-engineered dissolving microneedles for active transdermal delivery of nadroparin calcium. Eur J Pharm Biopharm 82: 299-307.

7. Gomaa YA, El-Khordagui LK, Garland MJ, Donnelly RF, Mclnnes F, et al. (2012) Effect of microneedle treatment on the skin permeation of a nanoencapsulated dye. J Pharm Pharmacol 64: 1592-1602.

8. Kalluri H, Banga AK (2011) Formation and closure of microchannels in skin following microporation. Pharm Res 28: 82-94

9. Gomaa YA, Morrow DI, Garland MJ, Donnelly RF, El-Khordagui LK, et al. (2010) Effects of microneedle length, density, insertion time and multiple applications on human skin barrier function: assessments by transepiderma water loss. Toxicol In Vitro 24: 1971-1978.

10. Nava-Arzaluz MG, Calderon-Lojero I, Quintanar-Guerrero D, Villalobos-Garcia R, Ganem-Quintanar A (2012) Microneedles as transdermal delivery systems: combination with other enhancing strategies. Curr Drug Deliv 9: 57-73.

11. van der Maaden K, Jiskoot W, Bouwstra J (2012) Microneedle technologies for (trans)dermal drug and vaccine delivery. J Control Release 161: 645-655.

12. Martanto W, Davis SP, Holiday NR, Wang J, Gill HS, et al. (2004) Transdermal delivery of insulin using microneedles in vivo. Pharm Res 21: 947-952.

13. Zhou CP, Liu YL, Wang HL, Zhang PX, Zhang JL (2010) Transdermal delivery of insulin using microneedle rollers in vivo. Int J Pharm 392: 127-133.

14. Qiu Y, Qin G, Zhang S, Wu Y, Xu B, et al. (2012) Novel lyophilized hydroge patches for convenient and effective administration of microneedle-mediated insulin delivery. Int J Pharm 437: 51-56. 
Citation: El-Khordagui LK (2012) Microneedles: An Emerging Approach for Active Transdermal Delivery of Insulin. J Bioequiv Availab 4: xxxi-xxxiii. doi:10.4172/jbb.10000e24

15. Chen H, Zhu H, Zheng J, Mou D, Wan J, et al. (2009) lontophoresis-driven penetration of nanovesicles through microneedle-induced skin microchannels for enhancing transdermal delivery of insulin. J Control Release 139: 63-72.

16. Qin G, Gao Y, Wu Y, Zhang S, Qiu Y, et al. (2012) Simultaneous basal-bolus delivery of fast-acting insulin and its significance in diabetes management. Nanomedicine 8: 221-227.

17. Daley TC, Prausnitz M, Felner E (2011) Insulin-coated microneedle arrays for bolus delivery to diabetic rats. Endocr Rev 32: P1-496.

18. Liu S, Jin MN, Quan YS, Kamiyama F, Katsumi H, et al. (2012) The development and characteristics of novel microneedle arrays fabricated from hyaluronic acid, and their application in the transdermal delivery of insulin. $\mathrm{J}$ Control Release 161: 933-941.
19. Ito Y, Hirono M, Fukushima K, Sugioka N, Takada K (2012) Two-layered dissolving microneedles formulated with intermediate-acting insulin. Int $J$ Pharm 436: 387-393

20. Harvey AJ, Kaestner SA, Sutter DE, Harvey NG, Mikszta JA, et al. (2011) Microneedle-based intradermal delivery enables rapid lymphatic uptake and distribution of protein drugs. Pharm Res 28: 107-116.

21. Gupta J, Felner EI, Prausnitz MR (2011) Rapid pharmacokinetics of intradermal insulin administered using microneedles in type 1 diabetes subjects. Diabetes Technol Ther 13: 451-456.

22. Khanna P, Strom JA, Malone JI, Bhansali S (2008) Microneedle-based automated therapy for diabetes mellitus. J Diabetes Sci Technol 2: 1122-1129. 